\title{
Prevalence of headache and migraine in schoolchildren
}

\author{
Ishaq Abu-Arefeh, George Russell
}

\begin{abstract}
Objectives-To determine the prevalence rates of the various causes of severe headache in schoolchildren, with special emphasis on migraine and its impact on school attendance.

Design-Population based study in two stages, comprising an initial screening questionnaire followed by clinical interviews and examination of children with symptoms and a control group of asymptomatic children matched for age and sex.

Setting -67 primary and secondary schools in the city of Aberdeen.

Subjects -2165 children, representing a random sample of $10 \%$ of schoolchildren in Aberdeen aged 5-15 years.
\end{abstract}

Main outcome measures-(a) the prevalence of migraine (International Headache Society criteria) and of other types of headache; (b) the impact of migraine on school attendance.

Results-The estimated prevalence rates of migraine and tension headache were $10.6 \%(95 \%$ confidence interval 9.1 to 12.3$)$ and $0.9 \%(0.5$ to 1.5$)$ respectively. The estimated prevalence rates for migraine without aura and migraine with aura were $7 \cdot 8 \%(95 \%$ confidence interval 6.5 to 9.3$)$ and $2.8 \%$ (2.0 to $3 \cdot 8)$ respectively. In addition, 10 children $(0.7 \%)$ had headaches which, though lasting less than two hours, also fulfilled the International Headache Society criteria for migraine, $14(0.9 \%)$ had tension headaches, and $20(1.3 \%)$ had non-specific recurrent headache. The prevalence of migraine increased with age, with male preponderance in children under 12 and female preponderance thereafter. Children with migraine lost a mean of 7.8 school days a year due to all illnesses $(2.8$ days (range $0-80$ ) due to headache) as compared with a mean of 3.7 days lost by controls.

Conclusions-Migraine is a common cause of headache in children and causes significantly reduced school attendance.

\section{Introduction}

Headache is a common symptom in paediatric hospital practice, the causes including migraine and tension headache as well as headache secondary to intracranial and extracranial infections, intracranial mass lesions, and head or neck trauma.' However, much less is known about the prevalence and causes of headache in non-hospital populations.

$B M F$ 1994:309:765-9
Migraine is said to be the most common cause of primary headache in children. ${ }^{2-4}$ The classic work of Bille on childhood headache and migraine in Sweden provided the first well conducted population based study. ${ }^{2}$ Bille, however, based his diagnosis of migraine on information obtained from a questionnaire and he interviewed only children in whom a diagnosis of "pronounced migraine" was based on the questionnaire responses. The $3.9 \%$ prevalence of migraine in his study must therefore be interpreted with caution. Other studies on the epidemiology of migraine in children have disclosed prevalence rates of between $2 \cdot 7 \%$ and $21.0 \%$ (table I). These studies have differed greatly in their methods of data collection, diagnostic criteria, age groups, and populations studied and have been concerned with either the lifelong or time specific prevalence of headache.

The diagnostic criteria for migraine defined by the International Headache Society ${ }^{5}$ are now widely accepted and have been applied successfully to studies on the epidemiology of migraine in adults. ${ }^{6-8} \mathrm{~A}$ recent study of childhood migraine in an urban general practice $^{9}$ showed minor differences in the prevalence of migraine according to the diagnostic criteria used. The rates were $3 \cdot 7 \%, 4 \cdot 2 \%$, and $4.9 \%$ for International Headache Society, Ad Hoc Committee, ${ }^{10}$ and Vahlquist ${ }^{11}$ criteria respectively. The study population, however, comprised 3-11 year old children from a single general practice and may not have been a true representation of the general childhood population.

The relation between childhood migraine and socioeconomic class has not been well documented. A study in a French adult population, however, found that migraine was commoner in the middle class than in the "executive" class ${ }^{6}$ whereas a study in the United States found that middle aged women from lower income households were at especially high risk. ${ }^{12}$

We thought that a population based study of childhood migraine was timely because (a) acceptable criteria for the diagnosis of migraine are now available ${ }^{5}$; (b) studies from the United States showed a significant increase in the prevalence of chronic migraine from $25.8 \%$ to $41.0 \%$ between 1980 and 1989 in the adult population, ${ }^{13}$ but no comparable paediatric data are available; $(c)$ previous large population based studies failed to include clinical interview as a prerequisite for the diagnosis of migraine and relied solely on information from questionnaires; and $(d)$ those studies that did base the diagnosis of migraine on clinical interview did not recruit from a large unselected childhood population extending over a wide age range.

This study was designed to assess the prevalence of migraine in a random sample of the entire childhood population of a single city, employing the International Headache Society criteria and validating the questionnaire responses with clinical interviews.

\section{Population and methods}

A total of 2165 children ( $10 \%$ of $5-15$ year olds attending 67 schools in the city of Aberdeen) were selected at random from the school lists without prior knowledge of their health or social background. Secondary schools and their feeder primary schools were studied sequentially. We did not approach the 
$6 \%$ of Aberdeen city children attending private schools.

The questionnaire, together with an explanatory letter, was issued to the selected children to take home for completion with the help of their parents. The questionnaire recorded the child's date of birth, sex, address, and the date of completing the questionnaire. The questions related to headache are listed in box $\mathrm{A}$.

$\begin{aligned} & \text { Box A-Questions about headache included in } \\ & \text { questionnaire }\end{aligned}$
- Has your child had headache
over the past year?
- If yes, were any of these
headaches severe enough to
stop normal activities?
- If yes, how many times has he
or she had severe headache in
the past year?
- Was there a cause for each
of these severe headaches?
- If yes, what was the cause for
each?

Children who did not return the questionnaire within two weeks were sent a reminder, posted to their home address.

Interviews-Children and their parents were invited to attend for clinical interview at the school medical room if their answers showed (a) that they had had at least two episodes of severe headache (defined as severe enough to interfere with normal activities) over the past year and $(b)$ that the headaches were not attributable to an infectious illness, trauma, or a specific illness (asthma, hay fever, medicines, and so on). For each child with symptoms so identified a control who did not have symptoms was identified, matching the symptomatic child for age and sex, and invited for interview. When neither parent could attend, the interview was conducted by telephone. Full details of the clinical history of the headache and past medical and family histories were obtained and the headaches classified into five groups (defined below). All children attending for interview had a full systematic physical examination, including blood pressure measurement. A detailed neurological examination including ophthalmoscopy was performed on children with headache.

Diagnosis-After interview and examination one of five diagnoses was made. Migraine was based on the criteria defined by the International Headache Society ${ }^{5}$ (box B). Migraine-like headache was diagnosed when the headache lasted less than two hours but otherwise fulfilled the International Headache Society criteria. Tension headache was diagnosed when the child had spells of daily headache for at least 10 days that was dull in quality, frontal or poorly localised, and not associated with nausea, vomiting, or light or noise intolerance and had behavioural or personality problems. ${ }^{3514}$ A specific diagnosis was made in a few children whose headaches could be explained on the basis of some other disorder, such as sinusitis. Nonspecific headache was diagnosed when none of the above categories applied.

Statistical analysis and estimation of prevalence-SPSS for Windows ${ }^{15}$ and confidence interval analysis ${ }^{16}$ were used. The estimated prevalence rates for migraine, migraine-like headache, tension headache, and nonspecific headache were calculated. The attendance rates for interview were almost identical $(85 \%)$ regardless of the cause to which the headache was attributed by the parents. It was therefore considered reasonable to adjust the observed prevalence rates to allow for the number of children with severe headache who failed to attend for interview, based on the assumption that the children who failed to attend had headaches in the same diagnostic categories and in the same proportion as those who did attend. Thus we estimated the prevalence rates of headache in the different age groups and diagnostic categories by multiplying the observed prevalence by a factor of $1 \cdot 17$, derived as follows: No of children invited for interview (241)/No who attended (206) $=1 \cdot 17$.

Ethics-The study was approved by the local ethics committee, the department of education, and the community child health service (school health service).

\section{Results}

QUESTIONNAIRES

Completed questionnaires were returned by 1754 children $(81 \%), 1601(74 \%)$ at the first request and 153 (27\% of non-respondents) after a reminder. The parents of 30 children declined to participate. Among the respondents there were 888 boys $(50.6 \%)$ and 866 girls $(49 \cdot 4 \%)$. Their mean age was 10.2 (SD 3.0 ) years. A total of 1166 children $(66 \%)$ had had headache over the past year, and in $391(22 \%)$ the episodes were severe enough to stop normal activities. The causes to which parents attributed severe headache are listed in table II.

\section{INTERVIEWS}

Two hundred and forty one children with severe recurrent headache, not attributed by the parents to infection, trauma, or specific illness (table III), were invited for clinical interview; 206 (85\%) attended within four to eight weeks of completing the questionnaire (mean six weeks). The parents of $186(90 \%)$ children either were present during the interviews (162) or gave interviews by telephone (24) as they were unable to attend with their children. Twenty children

Box B-Criteria for diagnosis of migraine (International Headache Society)

Migraine without aura

(A) At least five attacks fulfilling (B) to (D) below

(B) Headache lasting 4-72 hours (2-48 hours in children)

(C) Headache characterised by at least two of the following:

(1) Unilateral location

(2) Pulsating quality

(3) Moderate or severe intensity (inhibits or prohibits daily activities)

(4) Aggravated by climbing stairs or similar routine physical activity

(D) Headache accompanied by at least one of the following:

(1) Nausea or vomiting, or both

(2) Photophobia and phonophobia

Migraine with aura

(A) At least two attacks fulfilling (B) below

(B) Presence of at least three of the following:

(1) One or more fully reversible aura symptoms indicating focal cerebral cortical dysfunction or brain stem dysfunction, or both

(2) At least one aura symptom develops gradually over more than four minutes or two or more symptoms occur in succession

(3) No aura symptom lasts more than 60 minutes. When more than one aura symptom is present accepted duration is proportionally increased

(4) Headache follows aura with a free interval of less than 60 minutes. (It may also begin before or simultaneously with aura)
«Tiredness 3 , heat 3 , menstrual periods 2 , constipation 1 , poor sleep 2 , food 2. 
(10\%) gave interviews on their own. The attendance rate was $85 \%$ ( 50 out of 59 ) for children who attributed their headache to migraine and $86 \%$ ( 156 out of 182 ) for those who attributed their headache to other causes. After interview and examination the clinical diagnoses in the 206 children were as shown in table III.

TABLE III-Causes of severe headache diagnosed at interview and their prevalence rates

\begin{tabular}{lcc}
\hline Diagnosis & No (\%) of children & $\begin{array}{c}\text { Estimated } \\
\text { prevalence } \\
(\%)\end{array}$ \\
\hline Migraine (fulfilling criteria) & $159(77 \cdot 2)$ & $10 \cdot 6$ \\
Migraine-like headache (<2 hours) & $10(4 \cdot 9)$ & $0 \cdot 7$ \\
Tension headache & $14(6 \cdot 8)$ & 0.9 \\
Non-specific headache & $20(9 \cdot 7)$ & $1 \cdot 3$ \\
Specific diagnosis & $3(1 \cdot 5)^{\star}$ & $0 \cdot 2$ \\
\hline Total & $206(100 \cdot 0)$ & \\
\hline
\end{tabular}

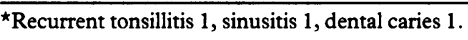

Among 50 children whose parents attributed the headache to migraine this diagnosis was confirmed in 45 ( $90 \%$ concordance). Of the remainder, four had non-specific headache and one migraine-like headache. None had tension headache. Of the 156 children whose headaches were attributed to causes other than migraine, $114(73 \%)$ had migraine, $9(6 \%)$ had migraine-like headache, 14 (9\%) had tension headache, $16(10 \%)$ had non-specific headache, and $3(2 \%)$ had other specific causes for their headaches.

The 153 children who returned the completed questionnaire after a reminder might be considered a proxy for the non-respondents. Twenty one $(14 \%)$ had severe recurrent headache and were invited to attend for interview. Sixteen $(10.5 \%)$ attended and were found to have migraine.

Each child fulfilling the diagnostic criteria for migraine was paired with an asymptomatic control matched for age and sex.

\section{PREVALENCE RATES}

The estimated prevalence rates were 10.6\% (95\% confidence interval $9 \cdot 1$ to $12 \cdot 3$ ) for migraine (International Headache Society criteria), $0.7 \%$ for migrainelike headache, $0.9 \%$ for tension headache, and $1.3 \%$ for non-specific headache (table III).

\section{CHILDREN WITH MIGRAINE}

Of the 159 children who fulfilled the diagnostic criteria for migraine, 74 were boys (mean age 11.2 (SD 2.7) years) and 85 girls (mean age $11.8(2.7)$ years). They represented the largest group of children with severe recurrent headache not associated with infection, trauma, or a specific condition. The estimated prevalence of migraine increased with age from $3.4 \%$ at age 5 years to a peak of $19 \cdot 1 \%$ at age 12 , then dropped slightly (table IV). A total of 117 children had migraine without aura (prevalence $7.8 \%$; 95\%

TABLE IV-Age and sex specific estimated prevalence rates of childhood migraine

\begin{tabular}{|c|c|c|c|c|c|}
\hline \multirow[b]{2}{*}{$\begin{array}{l}\text { Age } \\
\text { (years) }\end{array}$} & \multicolumn{2}{|r|}{ Boys } & \multicolumn{2}{|r|}{ Girls } & \multirow{2}{*}{$\begin{array}{c}\text { Total } \\
\text { prevalence } \\
(\%)\end{array}$} \\
\hline & $\begin{array}{c}\text { No } \\
\text { studied }\end{array}$ & $\begin{array}{c}\text { No of cases } \\
\text { (\% prevalence) }\end{array}$ & $\begin{array}{c}\text { No } \\
\text { studied }\end{array}$ & $\begin{array}{l}\text { No of cases } \\
\text { (\% prevalence) }\end{array}$ & \\
\hline 5 & 96 & $3(3 \cdot 7)$ & 78 & $2(3 \cdot 0)$ & $3 \cdot 4$ \\
\hline 6 & 86 & $5(6 \cdot 8)$ & 87 & $6(8 \cdot 1)$ & $7 \cdot 4$ \\
\hline 7 & 84 & $2(2 \cdot 8)$ & 85 & $3(4 \cdot 1)$ & 3.5 \\
\hline 8 & 96 & $7(8 \cdot 5)$ & 80 & $4(5.9)$ & $7 \cdot 3$ \\
\hline 9 & 88 & $5(6 \cdot 6)$ & 80 & $4(5.9)$ & $6 \cdot 3$ \\
\hline 10 & 72 & $7(11 \cdot 4)$ & 81 & $8(11 \cdot 6)$ & 11.5 \\
\hline 11 & 92 & $12(15 \cdot 3)$ & 103 & $10(11 \cdot 4)$ & $13 \cdot 2$ \\
\hline 12 & 87 & $15(20 \cdot 2)$ & 78 & $12(18 \cdot 0)$ & $19 \cdot 1$ \\
\hline 13 & 79 & $10(14 \cdot 8)$ & 69 & $14(23 \cdot 7)$ & $19 \cdot 0$ \\
\hline 14 & 58 & $2(4 \cdot 0)$ & 65 & $15(27 \cdot 0)$ & $16 \cdot 2$ \\
\hline 15 & 50 & $6(14 \cdot 0)$ & 60 & $7(13 \cdot 6)$ & $13 \cdot 8$ \\
\hline All & 888 & $74(9 \cdot 7)$ & 866 & $85(11 \cdot 5)$ & $10 \cdot 6$ \\
\hline
\end{tabular}

confidence interval 6.5 to 9.3 ) and 42 children had migraine with aura (prevalence $2 \cdot 8 \%, 2 \cdot 0$ to $3 \cdot 8$ ).

The overall sex distribution of children with migraine was almost equal $\left(\chi_{1}^{2}=1 \cdot 06 ; P=0 \cdot 3\right)$. However, in children aged 12 years or younger migraine was commoner in boys than in girls by a ratio of $1 \cdot 14: 1$ but in children aged over 12 migraine was commoner in girls by a ratio of $2 \cdot 0: 1 \quad\left(\chi_{1}^{2}=5 \cdot 73 ; P=0 \cdot 017\right)$. In children with migraine with aura there was a pronounced female preponderance ( 33 girls $v 9$ boys; $\chi_{1}^{2}=$ $13 \cdot 7, P=0 \cdot 0002$ ).

The social background of the children with migraine, as defined by parents' occupations, was comparable to those in the control group. Eleven per cent of children with migraine (17/159) were living with one parent (in 15 cases parents were divorced or separated, in two the father was dead) as compared with only $7 \%$ of their paired controls. This difference was not significant (McNemar's test; $P=0 \cdot 21$ ).

The personalities of the children with migraine were described by the parents or the children themselves as normal/happy in $113(71 \%)$ cases $(119(75 \%)$ controls), anxious in $12(8 \%)$ cases $(6(4 \%)$ controls), sensitive in $8(5 \%)$ cases (5 (3\%) controls), and moody in $26(16 \%)$ cases $(25$ (16\%) controls).

Children with migraine lost more school days than the controls due to any illness including headache (table $\mathrm{V}$ ), ranging from none to 82 days a year (mean paired differences $4 \cdot 1 \% ; 95 \%$ confidence interval $2 \cdot 4$ to 5.8). Even after excluding the days lost due to headache, children with migraine lost more school days due to other illnesses than the matched controls (mean paired difference $1 \cdot 3$; nil to $2 \cdot 6$ ).

TABLE V-Mean (range) number of days off school over one year

\begin{tabular}{|c|c|c|c|}
\hline $\begin{array}{l}\text { Reason for } \\
\text { time off school }\end{array}$ & Controls & $\begin{array}{l}\text { Children with } \\
\text { migraine }\end{array}$ & Paired $t$ test \\
\hline Migraine & 0 & $2 \cdot 8(0-80)$ & - \\
\hline Other illnesses & $3 \cdot 7(0-42)$ & $5 \cdot 0(0-49)$ & $P<0.05$ \\
\hline Total & $3.7(0.42)$ & $7 \cdot 8(0-82)$ & $P<0.0001$ \\
\hline
\end{tabular}

Twenty nine $(18 \cdot 2 \%)$ of the children with migraine also suffered from asthma as compared with $20(12 \cdot 6 \%)$ of the controls, but the difference was not significant (McNemar's test; $\chi_{1}^{2}=1 \cdot 561 ; \mathrm{P}=0 \cdot 211$ ).

\section{CHILDREN WITH MIGRAINE-LIKE HEADACHE}

Ten children fulfilled the criteria for migraine except that their attacks lasted less than two hours. Four children had unilateral headache, eight throbbing pain, eight experienced light and six noise intolerance, eight had anorexia, and seven had nausea. They represented $5 \%(10 / 206)$ of all children with recurrent severe headache (table VI).

TABLE VI-Features of other types of headache

\begin{tabular}{|c|c|c|c|c|c|}
\hline Type of headache & $\begin{array}{l}\text { No of } \\
\text { cases }\end{array}$ & $\begin{array}{l}\text { Boys: } \\
\text { girls }\end{array}$ & $\begin{array}{c}\text { Mean } \\
\text { age } \\
\text { (years) }\end{array}$ & $\begin{array}{c}\text { Mean } \\
\text { duration } \\
\text { (years) }\end{array}$ & $\begin{array}{c}\text { Estimated } \\
\text { prevalence } \\
(\%)\end{array}$ \\
\hline Migraine-like headache & 10 & $2 \cdot 3: 1$ & $11 \cdot 2$ & $3 \cdot 3$ & $0 \cdot 7$ \\
\hline Tension headache & 14 & $1 \cdot 3: 1$ & $9 \cdot 7$ & 2.5 & 0.9 \\
\hline Non-specific headache & 20 & $1 \cdot 5: 1$ & $9 \cdot 7$ & $1 \cdot 8$ & $1 \cdot 3$ \\
\hline
\end{tabular}

\section{ChILDREN With TENSION HEADACHE}

Fourteen children with recurrent severe headache had features characteristic of tension or psychogenic headache ${ }^{3514}$ (table VI). Children were described by their parents as shy, moody, anxious, depressed, or sensitive. Such personality traits are included in the definition of tension headache.

CHILDREN WITH NON-SPECIFIC HEADACHE

Twenty children had non-specific attacks of severe 
recurrent headache that could not be classified into either migraine or tension headache. The main feature of this group was the short history of headache (table VI).

\section{Discussion}

METHODOLOGY

We selected at random $10 \%$ of the children attending each school in order to ensure proportional representation of all areas of the city and, as far as possible, to cover all socioeconomic groups. We excluded children attending private schools in Aberdeen because they come from a much wider catchment area than the city itself and represent a small proportion of the total number of schoolchildren.

We believe that our two stage approach ensured accurate diagnostic classification of the headache and that by conducting the clinical interviews in the schools within eight weeks of completion of the questionnaire we ensured a high attendance rate and consistency of responses.

\section{ANALYSIS OF QUESTIONNAIRES AND INTERVIEWS}

The attendance rate at the interviews $(85 \%)$ was high enough to provide a reliable estimate of the prevalence of migraine in the general childhood population. Moreover, in the 153 children $(8.7 \%$ of the 1754 respondents) who returned completed questionnaires after a reminder the prevalence of migraine $(10.5 \%)$ was very close to the estimated prevalence in the whole population. Hence there was no evidence of a higher response rate from children with migraine.

The overall prevalence of all types of headache in the general childhood population was $66 \%$ (1166 cases in this series), increasing from $50 \%$ at age 5 years to around $80 \%$ at $12-15$ years. Our main aim, however, was to identify and categorise those children with severe recurrent headache that interfered with normal daily activities. The prevalence of severe headache showed only slight variation (20-25\%) across the age range, though the causes varied with age, severe headache due to infection being commoner in younger children and migraine being commoner in the older age group.

\section{PREVALENCE OF MIGRAINE}

Migraine was by far the most common cause of severe recurrent headache in the general childhood population. The estimated prevalence of $10.6 \%$ found in this study was more than twice the prevalence reported by Bille in $1962 .{ }^{2}$ However, the age and sex related trends were similar in both studies, there being a steady increase with age and a reversal of the sex distribution in favour of female preponderance in late childhood.

Our methodology differed from that of Bille, ${ }^{2}$ but probably the prevalence of migraine has risen over the past 30 years. A report from the United States disclosed a $60 \%$ increase in the prevalence of chronic migraine in an adult population between 1980 and 1989. ${ }^{13}$ Migraine has much in common with asthma and other atopic diseases in having an underlying genetic predisposition with attacks triggered by environmental and other physical factors. In our study the prevalence of asthma in children with migraine $(18 \cdot 2 \%)$ was higher than in controls $(12 \cdot 6 \%)$. Though not statistically significant, this difference is consistent with a report that the prevalence of asthma was twice as high in children of mothers with migraine than in children of mothers without migraine, ${ }^{17}$ suggesting that the two disorders are related genetically. A study from our department ${ }^{18}$ showed that the prevalence of asthma and other atopic diseases has roughly doubled in our childhood population over the past 25 years. It is highly unlikely that any significant change in the genetic constitution of the population could have occurred over such a short time and therefore it is probable that environmental changes are responsible.

SOCIAL AND PSYCHOLOGICAL FACTORS AND MIGRAINE

The social class distribution of the families of children with migraine did not differ significantly from that of the controls. Family breakdown was more frequent in association with migraine $(11 \%)$ than in the control group (7\%), but the difference was not significant. Larger studies may be necessary to assess the relation, if any, between childhood migraine and family breakdown. We did not carry out detailed psychological assessment of the children with migraine, but they had similar personalities to the control children as perceived by the children and their parents.

IMPACT OF MIGRAINE ON SCHOOL ATTENDANCE

The effects of migraine on children's education are difficult to assess in isolation from other confounding factors. Nevertheless, our data show a large number of school days were lost due to migraine, some children losing up to 80 days of schooling a year. It is also interesting that compared with controls children with migraine lost more days due to causes other than headache, suggesting that these children are vulnerable in other ways, possibly because of enhanced parental anxiety.

\section{APPLICABILITY OF HEADACHE SOCIETY CRITERIA}

In general the International Headache Society criteria for the diagnosis of migraine proved to be adequate in our study. Nevertheless, we found that restricting the diagnosis of migraine to children whose headaches lasted for at least two hours led to the exclusion of 10 children whose clinical features were so convincing that the diagnosis of migraine could not reasonably be excluded on duration alone. These children accounted for $6 \%$ of the cases of migraine. We believe that the minimal acceptable duration of migraine headache in children should be reduced from two hours to one hour to allow these children to be included.

\section{TENSION HEADACHE}

The prevalence of tension headache was in keeping with the observations of others, who find that tension headaches account for around $10 \%$ of all cases of severe recurrent headache in children. ${ }^{3}$ We based our definition of tension headache on clinical features derived from different sources. ${ }^{3514}$ Our estimated prevalence of tension headache $(0.9 \%)$ is, we believe, the first accurate report derived from a population based study and confirms the comparative rarity of the conditions as a cause of severe recurrent headache in children.

\section{NON-SPECIFIC HEADACHE}

Despite every effort to obtain full clinical details on all children with headache in our study we could not assign 20 cases to any identifiable group. These children generally suffered a shorter history of headache on average compared with the other groups. They may well be in an early stage of evolution of the migraine syndrome, but without long term observation it is impossible to assign them to any category.

\section{Conclusions}

We conclude that migraine is a common cause of headache in children, with a prevalence of about $11 \%$. It is an important cause of school absence. In children tension headache is much less common than migraine as a cause of headache severe enough to interfere with normal activities. Though no strictly comparable data 
Clinical implications

- Migraine is the most common cause of severe recurrent headache in children, affecting one child in nine between the ages of 5 and 15

- Migraine is an important cause of school absence

- Tension headache is an unusual cause of headache severe enough to interfere with normal activities

are available, comparison with other studies suggests that the prevalence of migraine in children may have risen over the past 30 years.

IAA was supported by a clinical fellowship from Glaxo Laboratories Limited. We are grateful to Mrs Daphne Russell for advice on statistical analysis, and to Mrs Connie Durno for help with data entry.

1 Hockaday JM. Migraine in children. Maternal and Child Health 1991;16: 246-7.

Bille B. Migraine in schoolchildren. Acta Paediatr 1962;51(suppl 136):1-151.

3 Barlow C. Psychogenic headache. In: Barlow CF, ed. Headache and migraine in childh. Pu

Hockaday JM. Definitions, clinical features and diagnosis of migraine. In Hockaday JM, ed. Migraine in childhood. London: Butterworth, 1988:5-24.

Hockaday JM, ed. Migraine in childhood. London: Butterworth, 1988:5-24.
Headache Classification Committee of the International Headache Society. Headache Classification Committee of the International Headache Society.
Classification and diagnostic criteria for headache disorders, neuralgias and facial pain. Cephalalgia 1988;8(suppl 7):1-96.

6 Henry P, Michel P, Brochet B, Dartigues JF, Tison S, Salamon R, et al. A nationwide survey of migraine in France: prevalence and clinical features in adults. Cephalalgia 1992;12:229-37.

7 Rasmussen BK, Jensen R, Schroll M, Olesen J. Epidemiology of headache in a general population-a prevalence study. I Clin Epidemiol 1991;44: $1147-57$.

8 Rasmussen BK, Olesen J. Migraine with aura and migraine without aura: an epidemiological study. Cephalalgia 1992;12:221-8.

9 Mortimer MJ, Kay J, Jaron A. Epidemiology of headache and childhood migraine in an urban general practice using ad hoc, Vahlquist and IHS criteria. Dev Med Child Neurol 1992;34:1095-101.

$10 \mathrm{Ad}$ Hoc Committee on the Classification of Headache. Classification of headache. FAMA 1962;179:717-8.

11 Vahlquist B, Hackzell G. Migraine of early onset: a study of thirty-one cases in which the disease first appeared between one and four years of age. Acta Paediatr 1949;38:622-36.

12 Stewart WF, Lipton RB, Celentano DD, Reed ML. Prevalence of migraine headache in the United States. Relation to age, income, race, and other sociodemographic factors. FAMA 1992;267:64-9.

13 Prevalence of chronic migraine headaches 1980-1989 in United States of America. Wkly Epidemiol Rec 1991;66:324-6.

14 Rossi LN, Cortinovis I, Bellettini G, Brunelli G, Bossi A. Diagnostic criteria for migraine and psychogenic headache in children. Dev Med Child Neurol 1992;34:516-23.

15 SPSS Inc. SPSS for Windows. Release 5.0.2. Chicago: SPSS, 1993.

16 Gardner SB, Winter PD, Gardner M. Confidence interval analysis version 1.0. London: British Medical Journal, 1989.

17 Chen TC, Leviton A. Asthma and eczema in children born to women with migraine. Arch Neurol 1990;47:1227-30.

18 Ninan TK, Russell G. Respiratory symptoms and atopy in Aberdeen schoolchildren: evidence from two surveys 25 years apart. BMF 1992;304: 873-5.

19 Dalsgaard-Nielsen T, Engberg-Pedersen H, Holm HE. Clinical and statistical investigations of the epidemiology of migraine. Dan Med Bull 1970;17: 138-48.

20 Green JE. Migraine in schoolchildren. Migraine News 1975 Sept:30

21 Deunber DC. An epidemiologic study of migraine and headache in 10-20 year olds. Headache 1977;17:173-80.

22 Sparks JP. The incidence of migraine in schoolchildren. Practitioner 1978;221: 407-11.

23 Sillanpaa $M$. Changes in the prevalence of migraine and other headaches during the first seven school years. Headache 1983;23:15-9.

(Accepted 28 fuly 1994)

\title{
Serum screening for Down's syndrome: experiences of obstetricians in England and Wales
}

\author{
Josephine M Green
}

\section{Abstract}

Objectives-To assess the experiences of obstetricians in England and Wales of serum screening for Down's syndrome.

Design-Postal questionnaire survey.

Subjects-Questionnaires were sent to all practising obstetricians in England and Wales with nonacademic appointments who had not participated in an earlier (randomly sampled) survey of obstetricians' attitudes $(n=555)$. Responses were received from 393 (71\%), of which 351 were analysed. The data represent about one third of obstetric consultants in Engand and Wales.

Main outcome measures-The extent of use of serum screening for Down's syndrome, and the problems encountered.

Results-Serum screening for Down's syndrome was being offered on some basis by virtually all obstetricians in the survey. Nearly half the sample said that they did not have adequate resources for counselling all the women to whom screening was offered. Many problems were reported, which in all cases were more common than equivalent problems encountered with serum screening for neural tube defects. Over $80 \%$ (289) said that women not understanding the test was a problem.

Conclusions-There is considerable confusion associated with serum screening for Down's syndrome. The precedent of serum screening for neural tube defects does not seem to have lessened the problems experienced, rather the contrary. Many obstetricians report inadequate resources for counselling, which is consistent with the high prevalence of problems associated with women not understanding the test. There is an urgent need to consider what counselling should consist of and who should undertake it and to ensure that necessary resources are available.

\section{Introduction}

Screening for fetal abnormality has become a major part of antenatal care, and there has been much discussion of the pros and cons of various testsnotably, serum screening for Down's syndrome. A survey in 1991 found that the use of such screening in the United Kingdom was expanding rapidly but in a piecemeal and sometimes inappropriate fashion. ${ }^{1}$ This survey was based on returns from directors of public health and asked about provision in 1991 and expected provision in 1992. Debates in the medical press suggest that many of those at the front of service provision are unhappy about the test, but there is no systematic information about the attitudes and experiences of obstetricians on this subject.

I carried out a survey of the attitudes of consultant obstetricians towards prenatal screening and diagnosis and towards termination of pregnancy. I report here on the questions relating to serum screening for Down's syndrome. Other aspects of the survey have been reported elsewhere ${ }^{2}$ and are being prepared for publication.

\section{Subjects and methods}

Names and addresses of members and fellows in England and Wales were obtained from the Royal College of Obstetricians and Gynaecologists. Subjects 